\title{
Synthesization of vortex beams by combining fork-shaped gratings for transmission electron microscopy
}

\author{
Ken Harada $^{1}$, Teruo Kohashi ${ }^{1}$ and Tomohiro Iwane ${ }^{1}$ \\ ${ }^{1}$ Central Research Laboratory, Hitachi Ltd., Hatoyama, Saitama 350-0395, JAPAN
}

Electron vortex beams are considered to be probes for next-generation electron beam devices because vortex beams carry intrinsic orbital angular momentum. Their use as probes should thus improve measurement capability [1, 2, 3].

We fabricated fork-shaped gratings with a 400 -nm lattice constant made from a $\mathrm{Si}_{3} \mathrm{~N}_{4}$ membrane with a 200-nm thickness using a focused ion beam machine (FB-2100, Hitachi High-Technologies Corp.). Electron diffractions from the gratings were observed using a prototype 300-kV field emission electron microscope [4] with an optical system constructed for small angle diffraction (camera length $150 \mathrm{~m}$ or larger) [5]. Ring-shaped spots in the observed diffraction patterns of the vortex beams were a typical feature. Furthermore, the shape and size of the grating openings were superimposed on the shape of the diffraction spots of the vortex beam [6].

A ring-shaped spot is directly usable as an incident beam probe for "scanning" type electron microscopes. For conventional transmission electron microscopes (TEMs), however, the feature of a spot is unusable because of its non-uniform irradiation density. To extend the use of vortex beam to TEMs, we are aiming at developing a plane-wave-like illumination probe with a uniform density. In the present work, we tried to superimpose several ring-shaped spots of different sizes to create uniform illumination in the reciprocal space. Three methods were investigated:

(1) superimpose vortex beams with different topological numbers,

(2) superimpose vortex beams with the same topological number but different diameters, and

(3) superimpose vortex beams with the same topological number but different diameters from a single phase grating.

Figure 1 shows the results for the second method. Nine fork-shaped gratings of third order dislocation with different diameters (10, 6.7, 3.3, and $1.7 \mu \mathrm{m})$ were combined as one grating-device (left panels), and diffraction patterns with superimposed ring-shaped diffraction spots (right panels). The rectangular lattice within the diffraction pattern in the right panels was caused by the four-fold symmetric arrangement of the gratings. This rectangular lattice, dot-like irradiation is undesirable for TEMs. We determined that random positioning of the gratings solved this problem.

Figure 2 shows the results for the third method. Single fork-shaped gratings of third order dislocation with two or three superimposed openings with different diameters are shown in the left panels, and their electron diffraction patterns are shown in the right panels. The phase of each grating lattice was a key factor in the harmonization of the superposition of the ring-shaped diffraction spots. With the third method, the grating was single phase, so the superposition was perfectly harmonized. However, the beam intensity for the smallest opening was too weak for it to be visible with the contrast in Fig. 2. 
The diffraction spots superimposed on the specimen were observed using small-angle electron diffraction when the specimen was positioned at the diffraction plane. These results show that the illumination area can be extended by using the second and third methods. The next step is to irradiate a specimen with a superimposed vortex beam and observe unprecedent objects, hopefully.

References:

[1] M. Uchida and A. Tonomura, Nature, 464, (2010) p. 737.

[2] J. Verbeeck et al., Nature, 469, (2010) p. 301.

[3] B. J. McMorran et al., Science, 331, (2011) p. 192.

[4] T. Kawasaki et al., Jpn. J. Appl. Phys., 29, (1990) p. L508.

[5] K. Harada, Appl. Phys. Lett., 100, (2012) p. 061901.

[6] K. Harada et al., Microsc. Microanal., 21, (2014) p. 274.

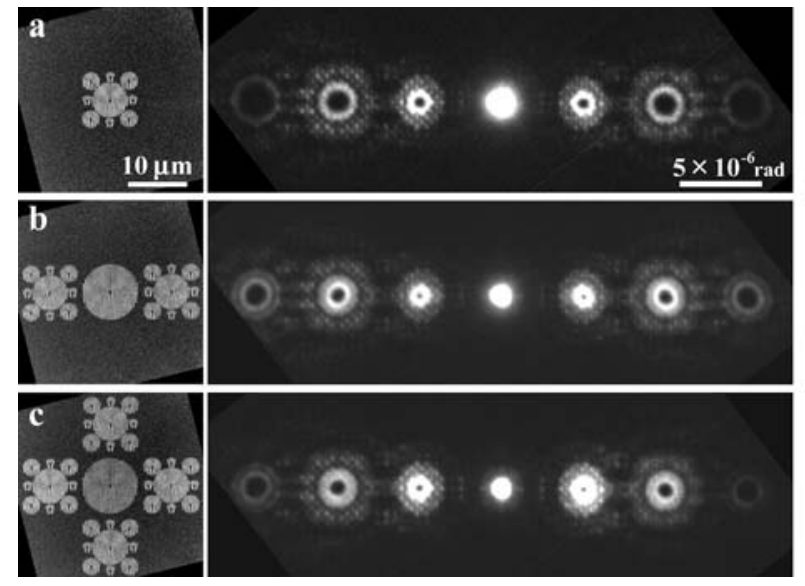

Figure 1.

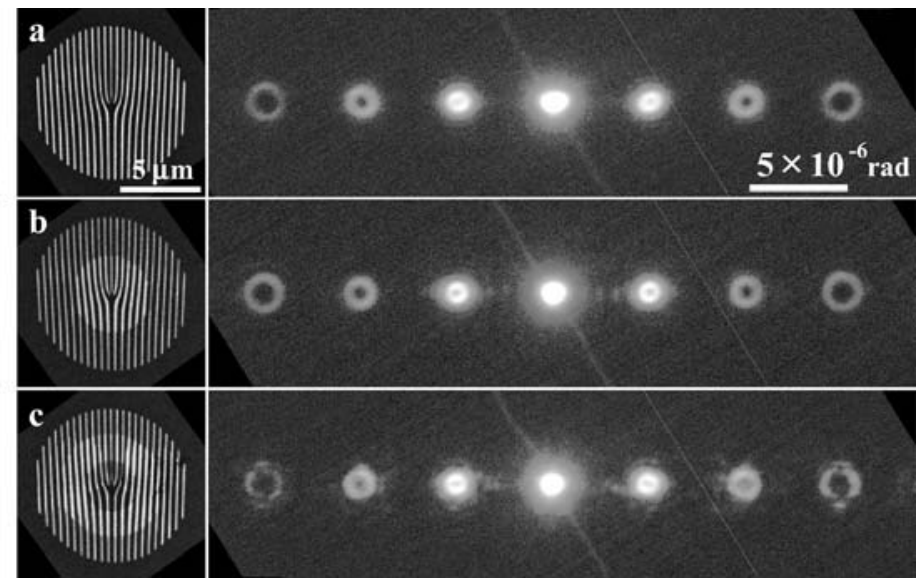

Figure 2.

Figure 1. Combined gratings as grating-devices (left) and electron diffraction patterns with superimposed ring-shaped diffraction spots (right): (a) single grating-device, (b) one grating and two grating-devices, (c) one grating and four grating-devices. Several rings were superimposed on diffraction pattern.

Figure 2. Fabricated single fork-shaped gratings of third order dislocation with two or three superimposed openings with different diameters (left) and electron diffraction patterns (right): (a) singlediameter grating, (b) double-diameter grating, (c) triple-diameter grating. 